\title{
Correspondence
}

\section{Bright red blood of Bhopal victims: cyanide or MIC?}

Sir,-Several anecdotal observations have contributed to the general perception of the nature of the toxic effects suffered by the victims of the Bhopal disaster.There have now been various reports of scientific investigations of the toxic effects of methyl isocyanate, the substance believed to have been released in the accident, and also various analyses of what conditions might have led to the explosive release of the substance. It has generally been found that symptoms observed in exposed people may be explained by what is now known of the toxicity of methyl isocyanate. ${ }^{12}$ On the other hand, some have claimed that other toxic compounds were involved, especially cyanides (organic or inorganic). So far as I am aware, no analytical data indicating the presence of such substances as a result of the accident have been presented. The observation of raised thiocyanate concentrations in the urine of both survivors and subsequent visitors to the region has been claimed as evidence of cyanide exposure. While this observation may well be accurate, it appears unlikely to be related to methyl isocyanate exposure on the basis of laboratory investigations of rats exposed to this substance. $^{12}$

One particular observation that has been quite widely quoted was made during postmortem studies immediately after the disaster. It was said that the blood (and possibly myoglobin) of victims of acute poisoning was bright red such as is usually associated with oxygenated (arterial) blood. This might be characteristic of cyanide poisoning where tissue uptake of oxygen is inhibited. On the other hand, it seems unlikely that human victims of methyl isocyanate poisoning would have had oxygenated blood: Nemery et al specifically measured oxygen levels in the blood of exposed rats and found them to be low, due to lung damage and bronchoconstriction impairing the exchange of blood gases. ${ }^{2}$ One might therefore conclude that some other substance was present either as a degradation product or impurity in the gas to which the Bhopal victims were exposed.

To evaluate this possibility we looked at the effect of methyl isocyanate on the ultraviolet/visible absorption spectrum of haemoglobin. ${ }^{3}$ A sample of blood obtained by heart puncture from a rat was diluted into $10 \mathrm{mM}$ sodium citrate buffer, $\mathrm{pH} 6.5$ : this was designed to produce a haemoglobin solution (by haemolysis of the erythrocytes) of suitable concentration for measurement of the absorption spectrum in a - Perkin Elmer 124 UV/vis spectrometer. Formation of methaemoglobin was inhibited by adding a small quantity of sodium dithionite. On addition of a small amount of methyl isocyanate $(20-50 \mu \mathrm{l}$ to $2 \mathrm{ml}$ solution) a vigorous reaction was observed in which gas evolved: this was the expected result of the hydrolysis reaction, the first stage of which generates methylamine and carbon dioxide. ${ }^{4}$ It was also observed that although the main Soret band absorption peak of haemoglobin (ca $430 \mathrm{~nm}$ ) was unaffected, the smaller peak in the visible region (ca $550 \mathrm{~nm}$ ) disappeared. A pale red colour remained in the solution representing the long tail of the ultraviolet peak. The same change was observed when a small amount of methylamine was added to a solution of haemoglobin. Both additions also caused a shift in $\mathrm{pH}$ of the solution towards the alkaline range (methylamine being quite strongly basic), but when sodium hydroxide was added to produce a similar shift no change in the spectrum was seen. Such spectral changes in haem proteins are by no means unexpected when molecules capable of acting as iron ligands are added: indeed, this is exactly the mechanism responsible for the colour difference between haemoglobin and oxyhaemoglobin. The spectroscopic change induced by methylamine is not identical with that produced by oxygenation. (Nor does it correspond to methaemoglobin formation, which would be prevented by dithionite: in fact, the presence of this compound had no effect on the modified spectrum.) It does seem possible, however, that the methylamine induced spectral change could be mistaken for this well known phenomenon by an observer using the naked eye, which is a crude tool for such tasks.

If this suggestion is accepted then the evidence for involvement of cyanide or other substances beside MIC, slim as it is, is further reduced.

A G SALMON

TUC Centenary Institute of Occupational Health, London School of Hygiene and Tropical Medicine, Keppel St, London WC1E 7HT.

\section{References}

${ }^{1}$ Salmon AG, Kerr-Muir M, Andersson N. Acute toxicity of methy isocyanate: a preliminary study of the dose response for eye and other effects. Br J Ind Med 1985;42:795-8.

${ }^{2}$ Nemery B, Dinsdale D, Sparrow S, Ray DE. Effects of methyl isocyanate on the respiratory tract of rats. $\mathrm{Br} J$ Ind Med 1985;42:799-805.

${ }^{3}$ Haurowitz F, Hardin R. Respiratory proteins. In: The proteins. Vol 2, part A. New York: Academic Press, 1954:296.

${ }^{4}$ Andersson N, Kerr Muir M, Salmon AG, et al. Bhopal disaster: eye follow-up and analytical chemistry. Lancet 1985;i:761-2. 